\title{
Dosimetric Evidences in Radioiodine Customized Hyperthyroidism Treatments
}

\author{
Angelo Ostinelli ${ }^{\star}$, Marta Duchini ${ }^{1}$, Giovanna Frigerio ${ }^{1}$, Angelo Corso ${ }^{2}$, Roberto Posterli ${ }^{2}$ and Marco Cacciatori ${ }^{1}$ \\ ${ }^{1}$ Department of Medical Physics, Ospedale Sant'Anna, Como, Italy \\ ${ }^{2}$ Department of Nuclear Medicine, Ospedale Sant'Anna, Como, Italy
}

\begin{abstract}
The radioiodine therapy is considered an almost definitive and successful hyperthyroidism treatment, alternative to surgery. Unlike a standardized activity approach is still adopted, a customized dosimetric study offers the significant advantage to take into account the individual variabilities in the structures to be treated. In the present work, some of the current issues relating to customized radioiodine treatment are discussed. The experience acquired during several years of customized hyperthyroidism radioiodine treatments performed at the S. Anna Hospital in Como (Italy) is presented, together with the main results of an extensive follow up analysis.
\end{abstract}

Keywords: Hyperthyroidism; Radiodiodine therapy; Outpatient residual activity; Treatment customization

\section{Introduction}

Hyperthyroidism and Graves' disease treatments require the administration of important ${ }^{131} \mathrm{I}$ activities [1-4] to achieve a nonhyperthyroidism status (i.e. euthyroidism or hypothyroidism) and cause significant patient residuals over the days following the radiopharmaceutical administration [5-9].

The prescription of standard therapeutic activities is still a common clinical practice. The standardized radioiodine activities range normally between 300 and $600 \mathrm{MBq} /$ patient, depending only on the thyroid volume, and they can exceed $1 \mathrm{GBq}$ for serious illnesses.

Otherwise, the customized treatments, based on in vivo kinetic studies, represent a valid choice, allowing the optimization of the administrated activity [10-16]. This approach offers an overall reduction of both the patient dose and the consequent radiation protection concern, maintaining the same level of therapeutic effectiveness. The actual Italian guidelines $[6,17]$ suggest that, at the discharge, the patient residual activity should be less than $0.030 \mathrm{mSv} / \mathrm{h}$ (in terms of ambient dose equivalent rate), corresponding to a residual activity less than 600 MBq.

In this context, the evaluation of the optimization process, in terms of clinical outcome and radiation protection impact, is of great significance.

The present communication is devoted to these important topics and describes the main physical aspects in the activity assessment, involving pre-therapeutic uptake measurements and functional volume evaluations. The radioiodine treatment procedure adopted at the Sant'Anna Hospital is described and the experimental evidences supporting its clinical effectiveness are also summarized. Since the residual internal activities may be significant, a short consideration regarding the patient radiation protection is also discussed and an innovative method to estimated the radioiodine residual activity is proposed.

\section{Materials and Methods}

According to the AIMN-AIFM (Associazione Italiana di Medicina Nucleare - Associazione Italiana di Fisica in Medicina) guidelines [17], the patient-specific bio-kinetic is studied by administering a ${ }^{131}$ I sodiumiodide track activity (about $2 \mathrm{MBq}$ ) in the pre-treatment phase. The radioiodine kinetic is described by the uptake curve: the experimental data are acquired at 2, 24, 96 hours from the administration time, with an adjunctive point at 6 hours for Graves' diseases. The data were fitted by a bi-exponential mathematical function, according to the two compartment model pharmacokinetics of ${ }^{131} \mathrm{I}$, by means of a homemade automatic software (PROFit) [18].

The extrapolated functional parameters are: the uptake percentage maximum value $U_{\max }$ and the radiopharmaceutical effective half-life $T_{1 / 2-e f f}$

To calculate the activity to be administered, two additional parameters must be defined: the functional thyroid volume and the target prescribed dose.

The functional thyroid volume was evaluated by both tomographic SPECT (using ${ }^{99 \mathrm{~m}} \mathrm{Tc}$-pertecnetate) and CT images, acquired by a SIEMENS SYMBIA T integrated diagnostic system. Both images were combined by means of a 3-D fusion software, working independently from the acquisition modalities. An additional estimation of the functional volume was provided by a home-made software developed with MATLAB 2010b and based on the Recovering Iterative Thresholding Method (RIThM) [19].

The prescribed dose was assessed according to specific clinical protocols.

The customized activity is finally computed by applying the following equations $[12,17]$ :

$$
A=5,829 \cdot \frac{D \cdot m}{U_{\max } \cdot T 1 / 2 \text { eff }}
$$

(Basedow-Graves' disease).

*Corresponding author: Angelo Ostinelli, Department of Medical Physics, Sant'Anna Hospital, via Ravona-22020 San Fermo della Battaglia (CO), Italy, Tel: (+39) 031585 9062; Fax: (+39) 031.585.9826; E-mail: angelo.ostinelli@hsacomo.org

Received November 23, 2015; Accepted December 29, 2015; Published January 06,2016

Citation: Ostinelli A, Duchini M, Frigerio G, Corso A, Posterli R, et al. (2016) Dosimetric Evidences in Radioiodine Customized Hyperthyroidism Treatments. Nucl Med Radiat Ther 7’: 273. doi:10.4172/2155-9619.1000273

Copyright: ( 2016 Ostinelli A, et al. This is an open-access article distributed under the terms of the Creative Commons Attribution License, which permits unrestricted use, distribution, and reproduction in any medium, provided the original author and source are credited. 
(multinodular and uninodular pre-toxic and toxic goiter).

where $D$ is the prescribed dose, $m$ is the functional mass, $T_{1 / 2-e f f}$ the radiopharmaceutical effective half-life and $U_{\text {max }}$ the uptake percentage maximum value.

In this study, 151 patients treated at the Department of Nuclear Medicine of the S. Anna Hospital have been considered. The specific thyroid pathologies were: the Graves' disease (50.9\%), the multinodular $(16.6 \%)$ and the uninodular (32.5\%) pre-toxic and toxic goiter. The statistical analysis was carried out by both dosimetric and the clinical response data and performed by the SPSS.20 Statistics software (SPSS inc. Chicago, IL, USA).

The mathematical model to evaluate the residual activity is based on the relationship between the activity, the ambient dose equivalent rate $H^{*}(10)$, the gamma constant $\Gamma$ and the source-detector distance (d) [4]. To consider both the iodine distribution and the tissue uptake, an effective distance $d_{\text {eff }}$ was introduced, where $d_{\text {eff }}=d+d_{x}$, where $d_{x}$ is virtual distance accounting for both the radiation source geometry and the body tissues attenuation. In this way, both the mean depth of the ${ }^{131}$ I $3 \mathrm{D}$ body distribution and the photon-tissues interaction are evaluated. For this aim, two measurements at known distances $\left(d_{1}\right.$ and $d_{2}$ ) are required. Applying the inverse square law to the $H^{\star}(10)$ rates and assuming that:

$$
C=\sqrt{\frac{\dot{H}_{2}^{*}(10)}{\dot{H} *_{1}(10)}}
$$

the mathematical relation between these variables becomes $d_{1+} d_{x=} \mathrm{C}$ $\left(d_{2+} d_{x}\right)$ and the activity can be calculated as:

$$
A=F^{2} \frac{\dot{H} *(10) d^{2}}{\tilde{A}}
$$

Where $F$ is expressed by the following relationship:

$$
F=\left(d+d_{x}\right) / d
$$

This algorithm was validated by a clinical trial including 40 patients, comparing the administered activities with the calculated ones. All these cases referred to customized radioiodine therapy: Graves' disease (19), uninodular (15) and multinodular toxic goiter (6). The external measurements were taken immediately after the radioiodine administration and before patient voiding (i.e. with the whole activity inside his/her body), at the hospital discharge (4 hours later) and during the follow-up (4 days later).

\section{Results}

The main outcome evidenced by the retrospective analysis of the pretherapeutic dosimetry clinical results is the mean reduction in the administered activities ( $435 \pm 150 \mathrm{MBq}$ /patient) compared to the standard one. These values represent a significant dose savings, given the consolidated tendency to use activities close to 500-600 MBq, which are approximately $20-25 \%$ higher than the customized ones.

The clinical response analysis pointed out that all uninodular and multinodular goiters recovered in a single treatment, while Graves' disease account for a $85.1 \%$ remission percentage. The low frequencies of Graves' disease re-treatments and the total remission of nodular goiters indicate that the customized radioiodine hyperthyroidism approach is very effective.

The proposed method to evaluate the residual activity provides an effective tool to check the radioiodine remainder at the patient discharge. The mean difference between administered and measured activities was about $0.5 \%$ and the standard deviation was $5.4 \%$. The mean percentage excreted activity was $11.3 \% \pm 12.7 \%$ at $3-4$ hours after the radioiodine administration, and $43.0 \% \pm 14.8 \%$, about 4 days later.

It seems evident that the absolute values of the excreted activity would be significantly higher in the case of standardized activity administration. The same clinical results confirm a significant dose reduction. As regard to the $d_{\text {eff }}$ extent, the mean reduction measured in the $d_{x}$ value (from $82 \pm 40 \mathrm{~mm}$ after the administration to $34 \pm 36 \mathrm{~mm}$ 4 day later) can be attributable to the progressive iodine uptake by the thyroid gland, which reduces both the tissues thickness crossed by the photons and the related attenuation.

\section{Discussion}

This study pointed out the excellent outcome of the treatment customization and the concomitant reduction of the administered activity, when compared to the standardized procedures.

On the basis of the theoretical-experimental model, a good procedure to estimate the residual activity and an effective radiation protection tool is offered, which significantly reduces the error margins within an approximate range of $\pm 11 \%$.

These results are more than enough to justify the efforts required to improve the procedures to customize the hyperthyroidism radioiodine treatments and to develop a check program to evaluate the residual activity of treated patients.

\section{References}

1. Eary JF, Brenner W (2007) Nuclear Medicine Therapy. Informa Healthcare New York London.

2. Salvatori M, Luster M (2010) Radioiodine therapy dosimetry in benign thyroid disease and differentiated thyroid carcinoma. Eur J Nucl Med Mol Imaging 37: 821-828.

3. Ross DS (2011) Radioiodine therapy for hyperthyroidism. N Engl J Med 364 $542-550$.

4. Stokkel MP, Handkiewicz Junak D, Lassmann M, Dietlein M, Luster M (2010) EANM procedure guidelines for therapy of benign thyroid disease. Eur $\mathrm{J}$ Nucl Med Mol Imaging 37: 2218-2228.

5. Marriott CJ, Webber CE, Gulenchyn KY (2007) Radiation exposure for 'caregivers' during high-dose outpatient radioiodine therapy. Radiat Prot Dosimetry 123: 62-67.

6. De Crescenzo S, Fattori S, Fioroni S, Indovina F, Pedroli G (2014) Raccomandazioni per la dimissione dei pazienti a seguito di terapia medico nucleare con 131l al fine della protezione contro i rischi da radiazioni ionizzanti. AIFM.

7. International Commission on Radiological Protection (2004) Release of patients after therapy with unsealed radionuclides. Ann ICRP 34: v-vi, 1-79.

8. NCRP (2006) Management of radionuclide therapy patients. Report: 155.

9. IAEA (2009) Release of patients after radionuclide therapy. Safety Reports Series: 63

10. Haase A, Bähre M, Lauer I, Meller B, Richter E (2000) Radioiodine therapy in Graves' hyperthyroidism: determination of individual optimum target dose. Exp Clin Endocrinol Diabetes 108: 133-137.

11. Di Martino F, Traino AC, Brill AB, Stabin MG, Lazzer M (2002) A theoretica model for prescription of the patient-specific therapeutic activity for radioiodine therapy of Graves' disease. Phys Med Biol 47: 1493-1499.

12. Traino A, Di Martino F, Lazzeri M (2004) A dosimetric approach to patientspecific radioiodine treatment of Graves' disease with incorporation of treatment induced changes in thyroid mass. Med Phys 31: 2121-2127.

13. Traino AC, Grosso M, Mariani G (2010) Possibility of limiting the unjustified irradiation in 131I therapy of Graves' disease: a thyroid mass reduction based method for the optimum activity calculation. Physica Medica 26: 71-79. 
Citation: Ostinelli A, Duchini M, Frigerio G, Corso A, Posterli R, et al. (2016) Dosimetric Evidences in Radioiodine Customized Hyperthyroidism Treatments. J Nucl Med Radiat Ther 7: 273. doi:10.4172/2155-9619.1000273

14. van Isselt JW, de Klerk JM, van Rijk PP, van Gils AP, Polman LJ, et al. (2003) Comparison of methods for thyroid volume estimation in patients with Graves disease. Eur J Nucl Med Mol Imaging 30: 525-531.

15. de Rooij A, Vandenbroucke JP, Smit JWA, Stokkel MPM, Dekkers OM (2009) Clinical outcomes after estimated versus calculated activity of radioiodine for the treatment of hyperthyroidism: systematic review and meta-analysis. Eur Journ of Endocrinol 161: 771-777.

16. Lassmann M, Chiesa C, Flux G, Bardiès M; EANM Dosimetry Committee (2011) EANM Dosimetry Committee guidance document: good practice of clinical dosimetry reporting. Eur J Nucl Med Mol Imaging 38: 192-200.
17. Dottorini ME, Inglese E, Salvatori M, Signore A, Squatrito S, et al. (2004) II trattamento radio metabolico dell'ipertiroidismo. Linee guida SIE-AIMN-AIFM SIA-AIMN-AIFM.

18. Cacciatori M, Coppi E, Ostinelli A (2003) Profit: uno strumento per il calcolo dell'attività nei trattamenti radiometabolici con 131I. Fisica in Medicina: 4.

19. Pacilio M, Basile C, Shcherbinin S, Caselli F, Ventroni G, et al. (2011) An innovative iterative thresholding algorithm for tumour segmentation and volumetric quantification on SPECT images: Monte Carlo-based methodology and validation. Med Phys 38: 3050 . 